4 Jian Liu, ${ }^{\mathrm{a}, \mathrm{b} *}$ Luogen Liu, ${ }^{\mathrm{a}, \mathrm{b} *}$ Shinuan Zeng, ${ }^{\mathrm{a}, \mathrm{b}}$ Xiaobin Meng, ${ }^{\mathrm{c}}$ Nanfeng Lei, ${ }^{\mathrm{c}}$ Hai

5 Yang, ${ }^{\mathrm{c}}$ Runcai Li, ${ }^{\mathrm{a}, \mathrm{b}}$ Xuemin Guo ${ }^{\mathrm{a}, \mathrm{b}} \#$

6

7 a Institute of Human Virology, Zhongshan School of Medicine, Sun Yat-Sen University,

8 Guangzhou Guangdong, China

9

\section{interferon-stimulated gene product}

${ }^{2}$ Key Laboratory of Tropical Disease Control (Sun Yat-Sen University), Ministry of

Education, Guangzhou, Guangdong, China

${ }^{3}$ Meizhou People’s Hospital, Meizhou, Guangdong, China

Running Title: Activity and mechanism of L3HYPDH against EV71

\#Address correspondence to Xuemin Guo, email: xmguo2005@yahoo.com

*Jian Liu and Luogen Liu contributed equally to this work.

Keywords: Interferon-stimulated gene, Enterovirus 71, L3HYPDH, Antiviral activity

Word counts for the abstract: 150

Word counts for the text: 4198

\title{
Inhibition of EV71 replication by L3HYPDH, a newly identified
}




\section{ABSTRACT}

Up-regulation of interferon-stimulated genes (ISGs) is key to antiviral states mediated by interferon (IFN) but little is known about activity and underlying mechanisms of most ISGs against Enterovirus 71 (EV71). EV71 causes hand-foot-mouth disease in infants and occasionally severe neurological symptoms. Here we report that the product of $L 3 H Y P D H$, a newly identified ISG, inhibits the replication of EV71. This anti-EV71 activity was mapped to the C-terminal 60 amino acids region as well as the $\mathrm{N}$-terminal region spanning from amino acid position 61 to 120 of L3HYPDH protein. L3HYPDH was shown to interfere with EV71 propagation at the RNA replication and protein translation levels. Specifically, L3HYPDH impairs translation mediated by the EV71 international ribosome entry site (IRES) but not by the HCV IRES, and this activity is conferred by the C-terminal region of L3HYPDH. Thus, L3HYPDH has antiviral activity against EV71, suggesting a potential mechanism for broad-spectrum antiviral effects of IFN. 


\section{IMPORTANCE}

Human EV71 can cause hand-foot-mouth disease (HFMD) and even death; however, no effective anti-EV71 treatment is available. Although EV71 suppresses induction of IFN and activation of IFN signaling pathways, type I IFN treatment can enhance the anti-EV71 state. IFN-stimulated genes (ISGs) are critical for innate immune defenses; however, the antiviral activities of many ISGs are not known. EV71 is seldom used for ISGs studies. So understanding the mechanism by which ISGs exert activity against EV71 will help to better understand IFN-triggered antiviral activity and provide new strategies to treat enterovirus infection. L3HYPDH is a newly identified ISG. We report here that L3HYPDH significantly inhibits EV71 replication by repressing RNA replication and protein translation, which suggests a mechanism underlying type I IFN against EV71. This would assist with the development of novel therapeutics to treat HFMD. 


\section{INTRODUCTION}

Hand-foot-mouth disease (HFMD) is a common viral disease in infants and children across the Asian-Pacific region, characterized by fever, rash, and occasionally severe neurological symptoms $(1,2)$. Enterovirus 71 (EV71) is a major causative agent of HFMD. Different from many other viruses, EV71 suppresses induction of type I interferons (IFNs) and activation of IFN signaling pathways, and consequently, inhibits host anti-viral defenses (3-5). Nonetheless, EV71-infected cells still respond to type I IFN treatment and display an enhanced antiviral state. For example, in vitro studies showed that some type I IFNs, including IFN- $\alpha 4$, IFN- $\alpha 6$, IFN- $\alpha 14$ and IFN- $\alpha 16$, significantly reduced cytopathic effect (CPE) induced by EV71 infection (6). An IFN- $\alpha 2 b$ aerosol therapy has been used topically to treat HFMD (7). However, how IFNs suppress EV71 infection is not clear.

IFN-mediated antiviral mechanisms are diverse and complicated. Up-regulation of IFN-stimulated genes (ISGs) has shown to be critical to innate immune defenses against invading pathogens, so studies are underway to assess their functions and underlying mechanisms for developing future antiviral therapies. ISGs are abundant and different cells respond variously to different types of IFNs and the stimulation duration, resulting in diverse ISG expression patterns (8-10). Data of several systematic detections for antiviral activity suggest that different sets of ISGs target different viruses in unique ways, and antiviral roles may be broad or specific, strong or weak $(11,12)$. However, EV71 is seldom used for characterizing antiviral activity of ISGs, including well-characterized classic ISGs. 
EV71 is enterovirus of the Picornaviridae family. Its genomic RNA is about 7400 nt long, single and positive-stranded, and contains only one open reading frame (ORF) flanked with a 5'-untranslated region (5'-UTR) and a 3'-UTR (1). The 5'-UTR contains a cloverleaf structure and an internal ribosome entry site (IRES), responsible for viral RNA replication and translation, respectively (13). The life cycle of EV71 starts with attachment to the host cell surface by recognition of a specific receptor, followed by endocytosis and release of viral RNA into the cytoplasm (14). Then, EV71 IRES initiates viral translation by recruiting host proteins. Synthesized polyproteins are processed into structural and non-structural proteins by its own protease 2A and 3C. When viral proteins accumulate, viral protein 3CD binds to the cloverleaf structure of 5'UTR to stop viral protein synthesis and initiates viral RNA replication. Produced RNAs then direct viral protein synthesis in large quantities. With the assembly of viral RNAs and proteins into virions, the host cell lyses and progeny viruses are released for a new round of infection $(15,16)$.

Many ISGs are antiviral effectors but these represent a few existing ISGs, and more will be identified. Recently, 91 new ISGs were identified from human immune cell lines after treatment with the consensus interferon (17), but their antiviral activity is unclear. Using a fluorescent activated cell sorting-based strategy for screening, we identified several ISGs with anti-EV71 efficacy (data not shown). One of them is C14orf149, which was identified as a gene encoding a trans-3-hydroxy-L-proline dehydratase and then renamed $L 3 H Y P D H$ (18). Here, we report that this ISG product, L3HYPDH, possesses antiviral activity against EV71, and its mechanism of action 
was investigated with a series of biochemical and genetic assays.

\section{MATERIALS AND METHODS}

\section{Plasmids construction}

pCAG-DsRed, a red fluorescent protein-expressing plasmid, has been described previously (19). pWSK-EV71-GFP is an infectious EV71-GFP cDNA clone, with a GFP-coding sequence inserted downstream of EV71 5'UTR and in frame fusion with the downstream VP4, and expression of EV71-GFP is driven by a T7 promoter (20). pcDNA3.1-T7RNP expresses T7 RNA polymerase. These plasmids were kindly provided by Dr. Liguo Zhang at the Institute of Biophysics, Chinese Academy of Sciences (IBP, CAS). A siRNA targeting the coding sequence of $L 3 H Y P D H$ from position 791 to 811 was designed according to the recommendation of Sigma-Aldrich (https://www.sigmaaldrich.com/catalog/genes) and named shRNA149. A pair of complementary oligonucleotides 5'-GATCCCCCAGATGAACAGGTTGACAGAATTCAAGAGATTCTGTCAACC TGTTCATCTGTTTTTA-3' (sense) and 5'-AGCTTAAAAACAGATGAACA GGTTGACAGAATCTCTTGAATTCTGTCAACCTGTTCATCTGGGG-3’

(antisense) were synthesized with 5' ends being BglII and HindIII restriction site overhangs. For each oligonucleotide, the target sequence was sense followed by antisense orientations separated by a nine-nucleotide spacer. Oligonucleotides were annealed and then cloned into the BglII and HindIII sites of pSUPER.retro.neo+gfp (Oligoengine, herein abbreviated for pSUPER-GFP) to generate 
XbaI, respectively, and inserted into similarly digested pcDNA4-To/myc-His B

(Invitrogen), resulting in pcDNA4-L3HYPDH, pcDNA4-L3HYPDH $\Delta$ N1, pcDNA4-L3HYPDH $\Delta$ N2, $\quad$ pcDNA4-L3HYPDH $\Delta$ N3, $\quad$ pcDNA4-L3HYPDH $\Delta \mathrm{C} 1$, pcDNA4-L3HYPDH $\Delta \mathrm{C} 2$, and pcDNA4-L3HYPDH $\Delta \mathrm{C} 3$. psiCHECK2-M was a modified form of psiCHECK-2 (Promega) with deletion of the HSV-TK promoter

(Fig 5A). Inverse PCR was performed with high-fidelity DNA polymerase Phusion (ThermoFisher) and a pair of back-to-back primers to amplify the whole plasmid except the HSV-TK promoter sequence. PCR products were self-ligated and resulted in psiCHECK2-M; meanwhile, a SalI and a NotI sites within the back-to-back primers were introduced into the plasmid. EV71-5'UTR and HCV-5'UTR were amplified

133 from pWSK-EV71-GFP and pNL4-3RL-HCV-FL (21) by PCR, respectively. After digestion with SalI and NotI, the PCR products were linked into the similarly digested psiCHECK2-M and resulted in psiCHECK2-M-EV71-5'UTR and psiCHECK2-M-HCV-5’UTR. All primers used are listed in Table S1.

\section{Cell culture and virus preparation}


141 and constitutively expresses the main EV71 receptor scavenger receptor class B

142 member 2 (SCARB2). To generate the cell line constitutively expressing tagged

143 L3HYPDH, 293A-SCARB2 cells were transfected with pcDNA4-L3HYPDH as

144 described below and selected with Zeocin $(200 \mu \mathrm{g} / \mathrm{ml})$. Resistant colonies were

145 individually expanded and detected by Western blot. One positive clone was chosen

146 and named 293A-SCARB2-L3HYPDH. This process was applied to the empty vector

147 and resulted in control cell 293A-SCARB2-Ctrl.

EV71-MZ (GenBank accession no. KY582572), isolated from the throat swab of

an ICU patient at Meizhou People’s Hospital in 2014, was amplified by successive

passages in RD cells until apparent CPE appeared. EV71-GFP was generated by

co-transfecting pWSK-EV71-GFP and pcDNA3.1-T7RNAP into 293A-SCARB2

cells as described previously (20). Viral supernatants were titrated using a plague assay, aliquoted, and then used for infection.

\section{Transfection and infection}

Depending on the experiments, cells were seeded into a 24-well or 6-well plate or

159 incubation for the indicated time, cells were treated as required. 
medium and incubated for specific time, followed by FACS assay, RT-qPCR measurement, or supernatant titration.

\section{Plaque assay}

The plaque assay was performed as described previously (22). Briefly, RD cells were incubated with viral supernatants undiluted or diluted in 10 -fold series for $1 \mathrm{~h}$. Subsequently, the supernatants were aspirated, and cells were covered with DMEM containing 1\% methylcellulose (Sigma-Aldrich) and 2\% FBS. After incubation for 4 days, cells were fixed with 4\% paraformaldehyde (Sigma-Aldrich) and stained with $0.1 \%$ crystal violet. Plaques were then quantified via visual scoring.

\section{Fluorescent activated cell sorting (FACS) assay}

To measure GFP production from EV71-GFP, $1 \times 10^{6}$ infected cells were collected and fixed in 4\% paraformaldehyde for 15 min. After washing three times with PBS, cells were resuspended in $0.5 \mathrm{ml}$ of PBS for flow cytometry (LSRFortessa, BD). To assess effects of $L 3 H Y P D H$ knockdown by RNAi, the cells were transfected with pSUPER-GFP-shRNA149 or pSUPER-GFP. After incubation for the indicated time, the cells were harvested and washed with PBS. GFP-positive cells were obtained through FACS, and then lysed for Western blot or seeded into a 24-well plate for EV71-GFP infection or reporter plasmid transfection as required.

\section{IFN stimulation}

Cells were treated with $1000 \mathrm{IU} / \mathrm{ml}$ of recombinant human IFN- $\alpha 2 \mathrm{~b}$ (Prospec) for different time, and then total RNAs were isolated and used to measure specific mRNA abundance by RT-qPCR. 


\section{In vitro transcription of EV71-GFP and microscope assay of GFP}

pWSK-EV71-GFP was linearized XbaI and EV71-GFP RNAs were transcribed

using the T7 RiboMax kit (Promega). After transfection into

293A-SCARB2-L3HYPDH and 293A-SCARB2-Ctrl cells, the GFP signal was

observed under a fluorescence microscope (System Microscope BX63, Olympus) at the indicated times; total RNAs were isolated for RT-qPCR assay.

\section{RNA isolation and RT-qPCR}

Total RNAs were isolated from cells using TRI Reagent (Sigma-Aldrich) according to the manufacturer's instructions. RT-qPCR was carried out as described previously (23) to measure target mRNA. Briefly, RNAs were treated with DNase using an RQ1 RNase-Free DNase Kit (Promega); cDNAs were synthesized using PrimeScript RT reagent Kit (Takara, Dalian) and then diluted and subjected to quantitative PCR using TransStart Green qPCR SuperMix (TransGen Biotch) in a CFX96 Touch Real-Time PCR Detection System (Bio-Rad). Primers used appear in Table S1.

\section{Immunofluorescence assay (IFA)}

Subcellular localization of L3HYPDH proteins and the attachment and endocytosis of EV71 virions were detected using IFA as described previously (24) with some modifications. Briefly, 293A-SCARB2-L3HYPDH and 293A-SCARB2-Crtl were individually seeded onto a coverslip. Polyclonal antibody (PAb) specific to c-myc (Sigma-Aldrich, 1:100) and Alexa Fluor 555-labeled anti-rabbit IgG (ThermoFisher, 1:100) were used as primary and secondary antibody, 
respectively, to localize the subcellular distribution of the tagged L3HYPDH proteins.

Similarly, 293A-SCARB2-L3HYPDH and 293A-SCARB2-Crtl cells were infected

with EV71-MZ (MOI, 100) at $4^{\circ} \mathrm{C}$ for $1 \mathrm{~h}$ to allow viral attachment or incubated for

IgG (ThermoFisher, 1:100) were used as primary and secondary antibody, respectively,

213 to visualize EV71 virions. Nuclei were stained with DAPI (Roche). Fluorescent

214 images of cells were captured using a Zeiss LSM780 META confocal imaging system.

\section{Western blot}

Western blot was performed as described previously (24) with some

221 (Sangon), followed by incubation with HRP-conjugated anti-mouse IgG and anti-rabbit IgG (Santa Cruz Biotechology), respectively. Proteins were visualized with ECL.

\section{Luciferase activity assay}


All the experiments involving counting or calculation were performed

Student's two-tailed $t$ test was used for statistical analysis by using GraphPad Prism significant.

\section{RESULTS}

\section{Over-expression of L3HYPDH inhibits EV71-GFP replication} using a FACS-based assay (Fig 1A). 293A-SCARB2 cells were used to facilitate

EV71 infection. After co-transfection with pCAG-DsRed and pcDNA4-L3HYPDH at a ratio of 1:3, the cells were infected with EV71-GFP. All DsRed-expressing cells were assumed to express L3HYPDH, and viral replication in the DsRed-positive populations was quantified by FACS assay of GFP at $18 \mathrm{~h}$ post infection (Fig 1B). replication.

\section{Expression of endogenous L3HYPDH suppresses EV71 replication}

The expression of endogenous $L 3 H Y P D H$ and its response to IFN- $\alpha 2 \mathrm{~b}$ treatment 
the basal mRNA level of L3HYPDH was slightly higher in 293A, 293A-SCARB2 and Vero cells than in RD and A549, and the lowest in Hela cells (Fig 2A). Upon exposure to IFN- $\alpha 2 b$, the level of L3HYPDH mRNA was up-regulated and peaked at about 18 $\mathrm{h}$ in 293A, 293A-SCARB2 and A549 and at about $12 \mathrm{~h}$ in Vero cells. Peak levels were 3 to 5 times higher than the basal level (Fig 2B). In contrast, the mRNA level changed little in Hela cells and even decreased a little in RD cells (Fig 2B). These results indicate that IFN- $\alpha 2 b$ stimulates the expression of L3HYPDH. However, the expression of L3HYPDH and its response to IFN- $\alpha 2 b$ differ in different cells, which is common for ISGs.

A shRNA specific to L3HYPDH, designated as shRNA149, was designed and transcribed from pSuper-GFP-shRNA149. Its knockdown efficiency was detected in 293A-SCARB2 cells by co-transfecting pcDNA4-L3HYPDH together with pSUPER-GFP-shRNA149 or with pSUPER-GFP as a control. Western blot analysis showed that the tagged L3HYPDH protein level decreased dramatically in the presence of shRNA149 (Fig 2C). To determine if the endogenous L3HYPDH could suppress EV71 replication, 293A-SCARB2 cells were transfected with pSUPER-GFP-shRNA149, and the GFP-positive cells were sorted by FACS, followed by EV71-MZ infection. RT-qPCR assay revealed that shRNA149 reduced L3HYPDH mRNA level by more than 80\% (Fig 2D) and increased EV71 mRNA level from 1 to 1.7 (Fig 2E), indicating that the expression of endogenous L3HYPDH impaired EV71 replication. In this way, L3HYPDH possesses antiviral activity and is involved in the inherent cellular suppression on EV71 replication. 


\section{L3HYPDH}

The region critical to L3HYPDH action against EV71 was mapped by serial 277 deletions combination with the FACS-based assay. Three N-terminal and three

278 C-terminal progressive deletion mutants of L3HYPDH are schematically shown in Fig 3 (middle panel), designated $\Delta \mathrm{N} 1, \Delta \mathrm{N} 2, \Delta \mathrm{N} 3, \Delta \mathrm{C} 1, \Delta \mathrm{C} 2$, and $\Delta \mathrm{C} 3$. Their coding sequences were cloned in fusion with a myc- $6 \times$ His tag at the C-terminus as with L3HYPDH WT. The resulting plasmids were individually transfected into 293A-SCARB2 cells together with pCAG-DsRed followed by EV71-GFP infection as described in Fig 1A. Western blot showed that the protein levels of these truncated mutants were somewhat lower than that of WT, but still comparable (Fig 3, lower panel). FACS assay showed that L3HYPDH $\triangle \mathrm{N} 2$ lacking the amino acids from position 1 to 120 significantly impaired the antiviral activity in comparison with WT, while L3HYPDH $\Delta$ N1 lacking the amino acids from position 1 to 60 only slightly weakened the antiviral activity. L3HYPDH $\Delta \mathrm{C} 1$ lacking the C-terminal 60 amino acids from 295 to 354 also weaken the antiviral activity somewhat, while further deletion did not heighten this impairment. These results indicate that the amino acid sequences from position 61 to 120 and from 295 to 354 are both required for the development of anti-EV71 activity of L3HYPDH.

EV71 replication is suppressed in the cell line 293A-SCARB2-L3HYPDH expressing L3HYPDH constitutively

To facilitate investigation of the antiviral mechanism, the cell line 293A-SCARB2- L3HYPDH expressing L3HYPDH constitutively and the 297 corresponding control cell line 293A-SCARB2-Ctrl were generated. These two cell lines were infected with EV71-GFP at an MOI of 0.1. FACS assay showed that the 
GFP production in 293A-SCARB2-L3HYPDH decreased to about $84 \%$ of control levels (Fig 4A). Upon infection with EV71-MZ, a clinical isolate of EV71, there was significantly less viral multiplication in 293A-SCARB2-L3HYPDH than in the control cell (Fig 4B). IFA showed that L3HYPDH proteins were mainly located in the cytoplasm (Fig 4C), consistent with its anti-EV71 action. Therefore, the cell 293A-SCARB2-L3HYPDH displays remarkable anti-EV71 activity due to the over-expression of L3HYPDH, and thus can be exploited to uncover the underlying antiviral mechanism.

\section{L3HYPDH interferes with the synthesis of viral RNA and proteins}

The effects of L3HYPDH on different life stages of EV71 replication were examined in 293A-SCARB2-L3HYPDH cells. Based on the knowledge that EV71 is only adsorbed on the cell surface and could not finish endocytosis at $4^{\circ} \mathrm{C}$, 293A-SCARB2-L3HYPDH and the control cells were incubated with EV71-MZ for 1 h at $4^{\circ} \mathrm{C}$ followed by IFA with the antibody specific to EV71 VP2. As shown in Fig 5A, massive number of virions distributed on the outer surfaces of both cell lines, showing no difference in numbers, indicating that L3HYPDH could not interfere with EV71 attachment. After attachment at $4^{\circ} \mathrm{C}$, the viruses were further incubated with the cells for an additional $30 \mathrm{~min}$ at $37^{\circ} \mathrm{C}$ to complete endocytosis. IFA showed that many viruses entered both cell lines, showing little difference (Fig 5B), indicating that L3HYPDH had no effect on EV71 endocytosis. In this way, these results demonstrate that L3HYPDH does not impede the viral attachment and endocytosis.

The effects of L3HYPDH on the synthesis of viral RNA and proteins were investigated by monitoring changes in their levels over time. 293A-SCARB2-L3HYPDH and 293A-SCARB2-Ctrl cells were infected with 
EV71-MZ. Total RNAs were isolated at different times post-infection and the viral RNA abundance was measured by RT-qPCR. The increase in EV71 RNA levels over time in 293A-SCARB2-L3HYPDH cells was much lower than in the control cells (Fig 5C). Due to the tight cross-talk between viral translation and viral RNA synthesis,

327 these results suggested that L3HYPDH might inhibit the synthesis of viral RNA, proteins, or both. To further confirm this assumption, EV71-GFP RNAs were transfected into 293A-SCARB2- L3HYPDH and the control cell, and then the viral RNA and GFP proteins were measured and compared at different times after transfection. Microscope and RT-qPCR analyses showed that both the number of

332 GFP-positive cells and the viral RNA level were much lower in 293A-SCARB2-L3HYPDH cells than in the control cells (Fig 5D, 5E). These results provide more evidence that L3HYPDH might suppress viral RNA replication, viral protein synthesis, or both.

\section{L3HYPDH impairs the translation mediated by EV71-5’UTR}

The repression of L3HYPDH on viral protein synthesis was investigated using a bicistronic reporter system. As shown in Fig 6A, psiCHECK-2-based reporter plasmids were constructed with the HSV-TK promoter deleted to generate the control (psiCHECK2-M) or replaced with EV71-5'UTR or HCV-5'UTR, which contains EV71 IRES or HCV IRES, respectively. pcDNA4-L3HYPDH or the empty vector was transfected into 293A cells together with one of the three reporter plasmids at a ratio of 3:1, and then the luciferase activity and mRNA level were measured after incubation for $48 \mathrm{~h}$. For these reporters, the mRNA level ratio of Fluc/Rluc in L3HYPDH-overexpressed cells was equal to that in the empty vector-transfected cells as revealed by RT-qPCR (Fig 6B). However, the luciferase activity ratio (Fluc/Rluc) showed variability (Fig 6C). Whether L3HYPDH was over-expressed or not, the 
Fluc/Rluc ratio of the control reporter was extremely low due to the absence of IRES;

349 the ratio of the EV71-5’UTR-containing reporter reduced by $29 \%$ upon overexpression of L3HYPDH; while the ratio of the HCV-5’UTR-containing reporter changed little. We here proposed that L3HYPDH could specifically inhibit the

352 reporter translation mediated by EV71 IRES. RNAi assay further provided evidence 353 for this speculation. 293A-SCARB2-L3HYPDH cells were transfected with the 354 shRNA149-expressing plasmid or the empty vector. Then the GFP-positive cells were isolated and transfected with the reporter plasmids. Compared to the control cells, the Fluc/Rluc ratio of the EV71-5'UTR-containing reporter in 293A-SCARB2-L3HYPDH cells increased moderately upon L3HYPDH knockdown (Fig 6D).

Given that the amino acid sequence from position 61 to 120 and the C-terminal 60 amino acids together contribute to anti-EV71 activity, we examined whether these sequences were involved in the action of inhibiting the EV71 IRES-mediated translation. 293A cells were transfected with the plasmids expressing L3HYPDH WT or deletion mutants together with the EV71-5'UTR-containing bicistronic reporter plasmids. Reporter assay revealed that, compared to the empty vector, the expression of WT, $\Delta \mathrm{N} 1, \Delta \mathrm{N} 2$ and $\Delta \mathrm{N} 3$ reduced the Fluc/Rluc ratio by approximately $25 \%$ in 293A cells, consisting with the result shown above; while the expression of $\Delta \mathrm{C} 1, \Delta \mathrm{C} 2$, and $\Delta \mathrm{C} 3$ had little effect on the ratio by comparison with control cells (Fig 6E). In combination with the results shown in Fig 3, these data indicate that the C-terminal sequence containing 60 amino acids from 295 to 354 is required for L3HYPDH to inhibit EV71-IRES-mediated translation.

Altogether, these results indicate that L3HYPDH can specifically impair the translation initiated by EV71-5'UTR, and the C-terminal region is responsible for this 
inhibiting activity.

\section{DISCUSSION}

In this work, we report that the recently identified ISG product L3HYPDH has

377 antiviral activity against EV71 according to RNAi knockdown and over-expression experiments. Over-expression of L3HYPDH repressed GFP production of EV71-GFP

379

(Fig 1B, 4B) and caused significant inhibition of propagation of the clinical isolate EV71-MZ (Fig 4C). L3HYPDH knockdown increased EV71 mRNA in 293A-CARB2 cells (Fig 2E), highlighting that this gene is an important ISG with antiviral activity. Additionally, our data showed that IFN- $\alpha 2 b$ treatment was less effective against EV71 in cell culture when expression of L3HYPDH was depressed by RNAi (Fig S1). Therefore, L3HYPDH is key to antiviral activity of IFN- $\alpha 2 b$ against EV71. The potential activity of L3HYPDH against other viruses is not known. Given that different viruses are usually targeted by unique sets of ISGs (11), an extensive investigation on L3HYPDH will help to further elucidate the mechanism of IFN-mediated innate immunity against invading viruses.

Our data show that L3HYPDH may interfere with EV71 replication at post-entry stage (Fig 4). Bicistronic reporter assays confirmed that expression of L3HYPDH inhibited translation initiated by EV71 IRES (Fig 6B), however, the reporter protein was less reduced than EV71 RNA and virus-carrying GFP production during the first round of infection (Fig 1B, 4A, 5C-E). These inconsistences suggest that L3HYPDH hampers EV71 replication at steps other than translation. Although inhibition of viral 
RNA replication is likely, other potential effects on viral RNA stability, viral assembly and viral release cannot be excluded. Therefore, L3HYPDH inhibits EV71 replication at least at two levels, and these data are in agreement with previous studies indicating that many ISGs block viral replication at multiple stages of the viral life cycle (25-27). Considering that a range of proteins are involved in the viral RNA replication and translation process, we performed co-immunoprecipitation and tandem affinity purification combination mass spectrometry to screen for proteins interacting with L3HYPDH. Neither viral nor host proteins were identified (data not shown). These results suggest that the association of L3HYPDH proteins with other proteins should be transient or weak. L3HYPDH might also function by binding to the viral RNA directly; however, no known RNA-binding domains were predicted with online software (data not shown).

Viral translation is completely host cell-dependent. To maximize efficiency, different viruses evolved many strategies to facilitate selective translation of viral mRNAs over host transcripts (15, 28-30). Among these, the IRES-mediated translation initiation is necessary for picornavirus and hepacivirus to replicate $(13,31)$. Reporter assays showed that expression of L3HYPDH impaired initiation of translation mediated by EV71 IRES but not HCV IRES (Fig 6B, 6C). These two IRES differ in nucleotide length and structure as well as in host factors required for translation initiation and regulation (32). A potential target of L3HYPDH should be involved in EV71-5'UTR-mediated translation. Given that an ISG may interfere with different stages of different viral life cycles, whether L3HYPDH has the activity against HCV is unclear. Meanwhile, despite being present in all picornaviruses, IRES is diverse in length and structure and requires different host factors to function 
(33-35). Whether L3HYPDH can inhibit other genuses of picornavirus by interfering with IRES-mediated translation is not clear is not clear, but this is worthy of study.

L3HYPDH is a trans-3-hydroxy-L-proline dehydratase, and specifically catalyzes the dehydration of dietary trans-3-hydroxy-L-proline and from degradation of proteins such as collagen IV that contain it. This dehydratase contains two active sites, a Cys residue at the 104 position and a Thr residue at the 273 position (18). Interestingly, the region required for anti-EV71 activity was mainly mapped to the amino acid sequence from position 61 to 120 of L3HYPDH protein (Fig 3), which contains the Cys104 active site. Whether this proline dehydratase activity is involved in the anti-EV71 activity is not known. L3HYPDH functions as an anti-EV71 effector. Understanding ISG products and antiviral spectra, as well as their mechanisms of action and biological function will help create novel therapeutics for HFMD.

\section{ACKNOWLEDGEMENTS}

This work was supported by the grants from Guangdong Innovative Research Team Program (2009010058), the National Key Program for Infectious Disease of China (2012ZX10001003), and Science and Technology Planning Project of Guangdong Province (A2016467 to X. Meng). We thank Dr. Liguo Zhang for providing the plasmids pCAG-DsRed, pWSK-EV71-GFP, pcDNA3.1-T7RNP and the cell line 293A-SCARB2. We also thank Dr. Guangxia Gao for providing the plasmids pLPCX-C14orf149 and pNL4-3RL-HCV-FL.

\section{REFERENCES}

1. Brown BA, Oberste MS, Alexander JP, Jr., Kennett ML, Pallansch MA. 1999. Molecular epidemiology and evolution of enterovirus 71 strains isolated from 
1970 to 1998. J Virol 73:9969-9975.

2. Mcminn PC. 2002. An overview of the evolution of enterovirus 71 and its clinical and public health significance. FEMS Microbiol Rev 26:91-107.

3. Lei X, Liu X, Ma Y, Sun Z, Yang Y, Jin Q, He B, Wang J. 2010. The 3C Protein of Enterovirus 71 Inhibits Retinoid Acid-Inducible Gene I-Mediated Interferon Regulatory Factor 3 Activation and Type I Interferon Responses. J Virol 84:8051-8061.

4. Lei X, Sun Z, Liu X, Jin Q, He B, Wang J. 2011. Cleavage of the adaptor protein TRIF by enterovirus 71 3C inhibits antiviral responses mediated by Toll-like receptor 3. J Virol 85:8811-8818.

5. Lu J, Yi L, Zhao J, Yu J, Chen Y, Lin MC, Kung HF, He ML. 2012. receptor 1. J Virol 86:3767-3776.

6. Yi L, He Y, Chen Y, Kung HF, He ML. 2011. Potent inhibition of human Enterovirus 71 disrupts interferon signaling by reducing the level of interferon enterovirus 71 replication by type I interferon subtypes. Antiviral Ther 16:51-58.

7. Lin H, Huang L, Zhou J, Lin K, Wang H, Xue X, Xia C. 2016. Efficacy and safety of interferon-alpha2b spray in the treatment of hand, foot, and mouth disease: a multicenter, randomized, double-blind trial. Arch Virol 161:3073-3080.

8. De Veer MJ, Holko M, Frevel M, Walker E, Der S, Paranjape JM, Silverman RH, Williams BRG. 2001. Functional classification of interferon-stimulated genes identified using microarrays. J Leukoc Biol 69:912-920. 
differentially regulated by interferon $\alpha, \beta$, or $\gamma$ using oligonucleotide arrays. Proc Natl Acad Sci U S A 95:15623-15628.

10. Liu SY, Sanchez DJ, Aliyari R, Lu S, Cheng G. 2012. Systematic identification of type I and type II interferon-induced antiviral factors. Proc Natl Acad Sci U S A 109:4239-4244.

11. Schoggins JW, Rice CM. 2011. Interferon-stimulated genes and their antiviral effector functions. Curr Opin Virol 1:519-525.

12. Schneider WM, Chevillotte MD, Rice CM. 2014. Interferon-stimulated genes: a complex web of host defenses. Annu Rev Immunol 32:513-545.

13. Martínez-Salas E, Francisco-Velilla R, Fernandez-Chamorro J, Lozano G, Diaz-Toledano R. 2015. Picornavirus IRES elements: RNA structure and host protein interactions. Virus Res 206:62-73.

14. Dang M, Wang X, Wang Q, Wang Y, Lin J, Sun Y, Li X, Zhang L, Lou Z, Wang J, Rao Z. 2014. Molecular mechanism of SCARB2-mediated attachment and uncoating of EV71. Protein Cell 5:692-703.

15. Lin JY, Chen TC, Weng KF, Chang SC, Chen LL, Shih SR. 2009. Viral and host proteins involved in picornavirus life cycle. J Biomed Sci 16:103.

16. Lei X, Cui S, Zhao Z, Wang J. 2015. Etiology, pathogenesis, antivirals and vaccines of hand, foot, and mouth disease. Natl Sci Rev 2:268-284.

17. Zhang X, Yang W, Wang X, Zhang X, Tian H, Deng H, Zhang L, Gao G. 2018. Identification of new type I interferon-stimulated genes and investigation of their involvement in IFN-beta activation. Protein Cell 1-9. 
488

489

490

491

492

493

494

495

496

497

498

499

500

501

502

503

504

505

506

507

508

509

18. Visser WF, Verhoeven-Duif NM, de Koning TJ. 2012. Identification of a human trans-3-hydroxy-L-proline dehydratase, the first characterized member of a novel family of proline racemase-like enzymes. J Biol Chem 287:21654-21662.

19. Matsuda T, Cepko CL. 2004. Electroporation and RNA interference in the rodent retina in vivo and in vitro. Proc Natl Acad Sci U S A 101:16-22.

20. Zhang X, Yang P, Wang N, Zhang J, Li J, Guo H, Yin X, Rao Z, Wang X, Zhang L. 2017. The binding of a monoclonal antibody to the apical region of SCARB2 blocks EV71 infection. Protein Cell 8:590-600.

21. Zhu Y, Wang X, Goff SP, Gao G. 2012. Translational repression precedes and is required for ZAP-mediated mRNA decay. EMBO J 31:4236-4246.

22. Tang WF, Huang RT, Chien KY, Huang JY, Lau KS, Jheng JR, Chiu CH, Wu TY, Chen CY, Horng JT. 2015. Host miR-197 plays a negative regulatory role in the enterovirus 71 infectious cycle by targeting the RAN protein. J Virol 90:1424-1438.

23. Zhou S, Chen X, Meng X, Zhang G, Wang J, Zhou D, Guo X. 2015. "Roar" of blaNDM-1 and "silence" of blaOXA-58 co-exist in Acinetobacter pittii. Sci Rep 5:8976.

24. Li K, Zhou S, Guo Q, Chen X, Lai DH, Lun ZR, Guo X. 2017. The eIF3 complex of Trypanosoma brucei: composition conservation does not imply the conservation of structural assembly and subunits function. RNA 23:333-345.

25. Pichlmair A, Lassnig C, Eberle CA, Gorna MW, Baumann CL, Burkard TR, Burckstummer T, Stefanovic A, Krieger S, Bennett KL, Rulicke T, Weber F, 
Colinge J, Muller M, Superti-Furga G. 2011. IFIT1 is an antiviral protein that recognizes 5'-triphosphate RNA. Nat Immunol 12:624-630.

26. Kato H, Takeuchi O, Sato S, Yoneyama M, Yamamoto M, Matsui K, Uematsu S, Jung A, Kawai T, Ishii KJ. 2006. Differential roles of MDA5 and RIG-I helicases in the recognition of RNA viruses. Nature 441:101-105.

27. Haller O, Kochs G. 2011. Human MxA protein: an interferon-induced dynamin-like GTPase with broad antiviral activity. J Interferon Cytokine Res 31:79-81.

518

28. Du H, Yin P, Yang X, Zhang L, Jin Q, Zhu G. 2014. Enterovirus 71 2C Protein Inhibits NF-אB Activation by Binding to RelA(p65). Sci Rep 5:14302.

29. Sharma R, Raychaudhuri S, Dasgupta A. 2004. Nuclear entry of poliovirus protease-polymerase precursor 3CD: implications for host cell transcription shut-off. Virology 320:195-205.

30. Weng KF, Li ML, Hung CT, Shih SR. 2009. Enterovirus 71 3C Protease Cleaves a Novel Target CstF-64 and Inhibits Cellular Polyadenylation. PLoS Pathog 5:e1000593.

31. Honda M, Ping LH, Rijnbrand RC, Amphlett E, Clarke B, Rowlands D, Lemon SM. 1996. Structural requirements for initiation of translation by internal ribosome entry within genome-length hepatitis C virus RNA. Virology 222:31-42.

32. Michael Gale J, Tan SL, Katze MG. 2000. Translational Control of Viral Gene Expression in Eukaryotes. Microbiol Mol Biol Rev 64:239-280. 
33. Gingras AC, Svitkin Y, Belsham GJ, Pause A, Sonenberg N. 1996. Activation of the Translational Suppressor 4E-BP1 Following Infection with Encephalomyocarditis Virus and Poliovirus. Proc Natl Acad Sci U S A 93:5578-5583.

34. Andreev DE, Fernandezmiragall O, Ramajo J, Dmitriev SE, Terenin IM, Martinezsalas E, Shatsky IN. 2007. Differential factor requirement to assemble translation initiation complexes at the alternative start codons of foot-and-mouth disease virus RNA. RNA 13:1366-1374.

35. Sweeney TR, Abaeva IS, Pestova TV, Hellen CU. 2014. The mechanism of translation initiation on Type 1 picornavirus IRESs. EMBO J 33:76-92. 


\section{Figure Legends}

\section{Fig 1. FACS-based assay for antiviral activity of L3HYPDH against EV71-GFP}

replication. (A) Overview of the procedures detecting anti-EV71 activity of over-expressed L3HYPDH using FACS. (B) FACS plots of L3HYPDH inhibition of EV71-GFP in 293A-SCARB2 cells. Numerals represent percent of total cell counts.

(C) GFP production, which was calculated by multiplying GFP and DsRed co-positive cell number by the mean value of GPF intensity. The value of the control cells transfected with empty vector was set as $100 \%$. Results are represented as means \pm SD of three independent experiments. ${ }^{*}, P<0.05$.

Fig 2. Anti-EV71 activity of the endogenous L3HYPDH. (A) RT-qPCR assay of the endogenous $L 3 H Y P D H$ mRNA level in different cell lines, which was normalized to GAPDH mRNA level. (B) RT-qPCR assay of $L 3 H Y P D H$ expression with IFN- $\alpha 2 b$ (1000 IU/ml) treatment for indicated time in different cell lines. L3HYPDH mRNA level was normalized to that of GAPDH. The relative mRNA level from untreated cells (marked as $0 \mathrm{~h}$ ) was set as 1 . (C) Western blot of knockdown efficiency of shRNA149 targeting L3HYPDH. 293A-SCARB2 cells were transfected with pcDNA4-L3HYPDH together with shRNA149-expressing plasmid or control plasmid (Crtl) at a ratio of 1:3.The GFP-positive cells were sorted and then infected with EV71, followed by RT-qPCR analyses of L3HYPDH mRNA (D) and EV71 2C mRNA after depression of L3HYPDH expression. RT-qPCR data are means \pm SD of three independent experiments. ${ }^{*}, P<0.05$.

\section{Fig 3. Mapping amino acid sequence required for anti-EV71 activity of}


L3HYPDH. Deletion mutants of L3HYPDH were schematically shown in the middle panel. Numbers indicate starting and ending amino acid. The plasmids expressing L3HYPDH wild type (WT) or truncated mutants were individually transfected into 293A-SCARB2 cells together with pCAG-DsREd at a ratio of 3:1, followed by EV71-GFP infection. Same performance was done the empty vector used as a control (Ctrl). L3HYPDH WT proteins or deletion mutants were analyzed by Western blot with anti-6×His MAb (lower panel). GFP production from EV71-GFP was detected using FACS and calculated as described in Fig1C. The value from control was set as $100 \%$. Data are represented as mean \pm SD of three independent experiments. ${ }^{*}, P<$ 0.05 .

Fig 4. Evaluating antiviral activity of 293A-SCARB2- L3HYPDH cells. 293A-SCARB2-L3HYPDH and the control cell 293A-SCARB2-Ctrl were infected with EV71-GFP (MOI, 0.1). GFP production was detected using FACS (A) and calculated (B). Data are represented as mean \pm SD of three independent experiments. *, $P<0.05$. (C) Time-viral yield assay. 293A-SCARB2-L3HYPDH and the control cell were individually infected with EV71-MZ (MOI, 2). The culture supernatants were harvested at different time points as indicated and titrated by plague assay. Data for each time point are means \pm SD of three independent experiments. (D) Subcellular localization of tagged L3HYPDH proteins using IFA. Nuclear DNA was stained with DAPI.

Fig 5. Stage assays for unveiling the mechanism of L3HYPDH against EV71. Effect of L3HYPDH on attachment (A) and endocytosis (B) of EV71 was examined using IFA. 293A-SCRB2-L3HYPDH and 293A-SCARB2-Ctrl cells were infected 

on viral RNA measured with RT-qPCR. 293A-SCARB2-L3HYPDH and control cell

591 (Ctrl) were infected with EV71-MZ (MOI, 2). EV71 2C mRNA level was measured at indicated times and normalized to that of $G A P D H$, with the relative level in control cell at $2 \mathrm{~h}$ post infection set as 1. EV71-GFP RNAs were transfected into 293A-SCARB2-L3HYPDH and control cell (Ctrl). GFP signal and EV71 2C RNA level at different times post transfection were examined by fluorescent microscope (D) and RT-qPCR (E), respectively. All the RT-qPCR data are represented as means \pm SD of three independent experiments. ${ }^{*}, P<0.05$.

Fig 6. Bicistronic reporter assay to measure the effect of L3HYPDH on EV71 IRES mediated translation. (A) Schematic of bicistronic reporters. Rluc is translated in a cap-dependent manner and Fluc in an IRES-dependent manner. pcDNA4-L3HYPDH or empty vector was transfected into 293A cells together with psiCHECK2-M, psiCHECK2-EV71-5'UTR, or psiCHECK2-HCV-5'UTR. Effects of L3HYPDH on the reporter expression were estimated by RT-qPCR (B) and luciferase activity assay (C). Fluc/Rluc ratio was calculated, with the relative value from the cells transfected with empty vectors was set as 1. (D) RNAi assay of effect of L3HYPDH on reporter expression mediated by IRES. pSUPER-GFP-shRNA149 or pSUPER-GFP was transfected into 293A-SCARB2-L3HYPDH cell. The GFP positive cells were transfected with psiCHECK2-M or psiCHECK2-M-EV71-5’UTR. Luciferase activity was measured, with Fluc/Rluc ratio from cells co-transfected with psiCHECK2-M and pSUPER-GFP set as 1. (E) Effect of L3HYPDH deletion on reporter expression. The plasmids expressing L3HYPDH WT or deletion mutants were individually transfected into 293A cells together with psiCHECK2-EV71-5'UTR at a ratio of 3:1. Transfection with pcDNA4 was used as a 
bioRxiv preprint doi: https://doi.org/10.1101/304345; this version posted April 19, 2018. The copyright holder for this preprint (which was not certified by peer review) is the author/funder, who has granted bioRxiv a license to display the preprint in perpetuity. It is made available under aCC-BY-NC-ND 4.0 International license.

control (Ctrl). Luciferase activities were measured. Fluc/Rluc ratio was calculated,

615 with the relative value from the cells transfected with the empty vector set as 1 . Data

616 are means \pm SD of three independent experiments. ${ }^{*}, P<0.05$. 


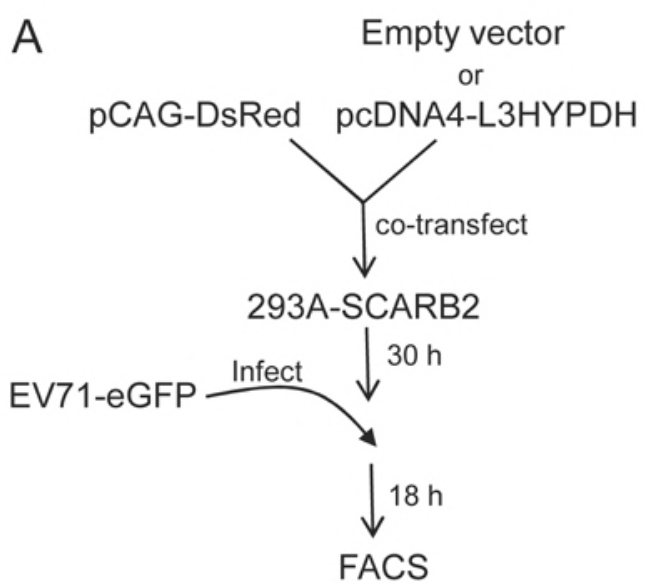

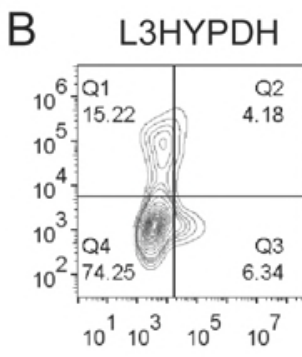

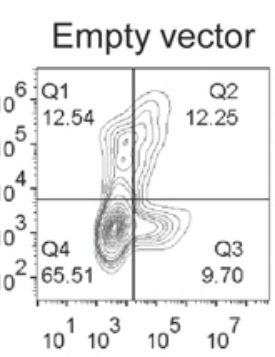

Un-transfected
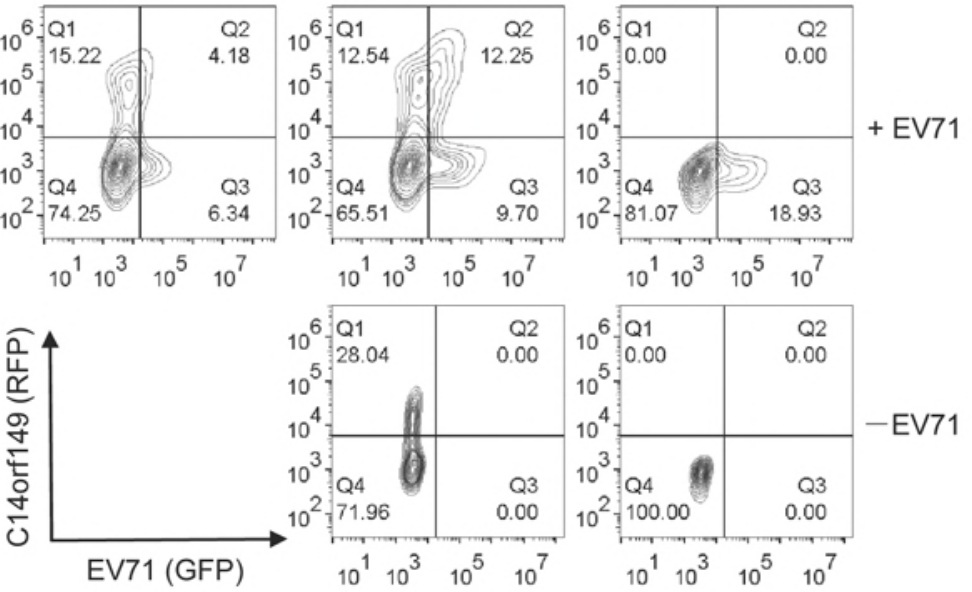

$10^{1} \quad 10^{3} \quad 10^{5} \quad 10^{7}$
C

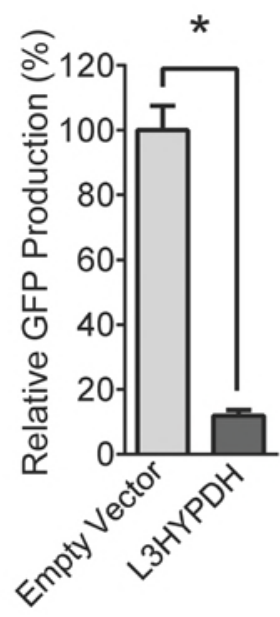



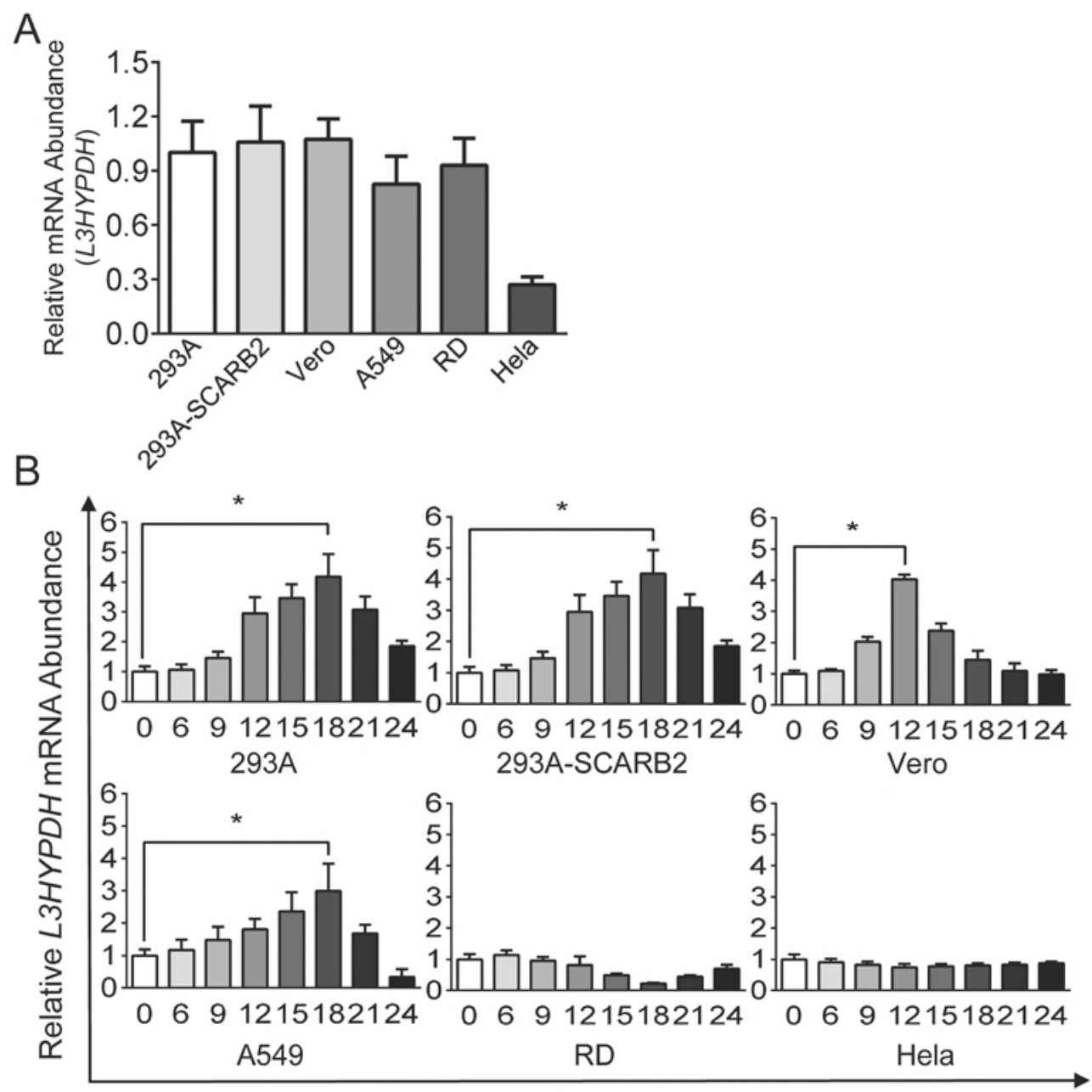

Time after IFN- $\alpha 2 b$ addition $(\mathrm{h})$
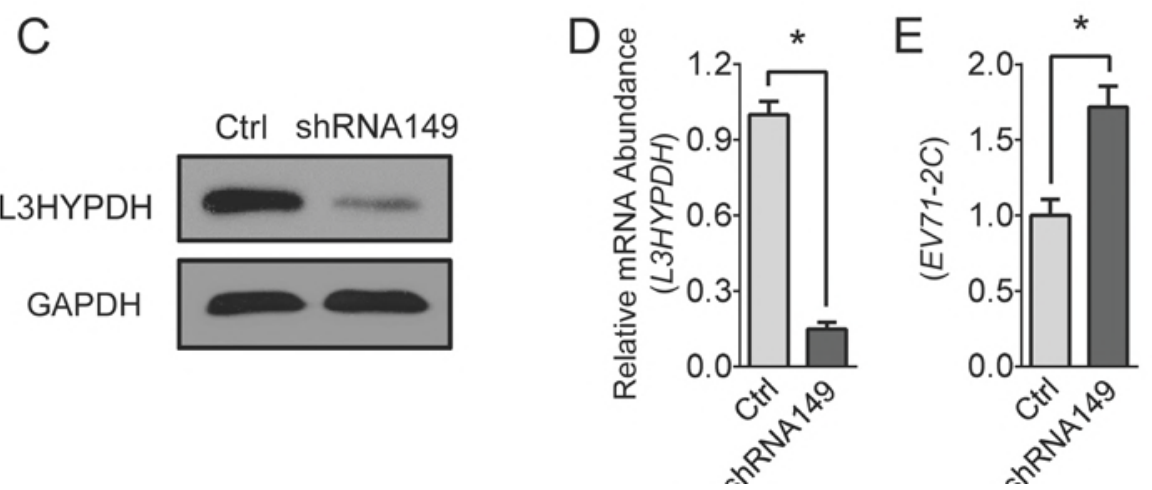


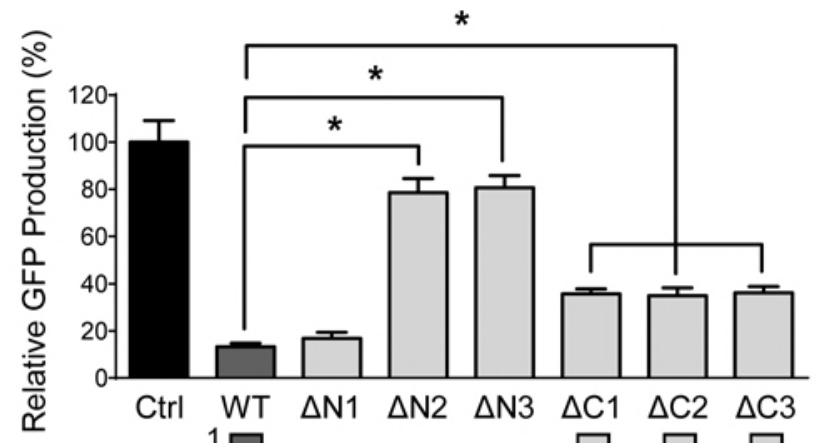

WB:

anti- $6 \times$ His

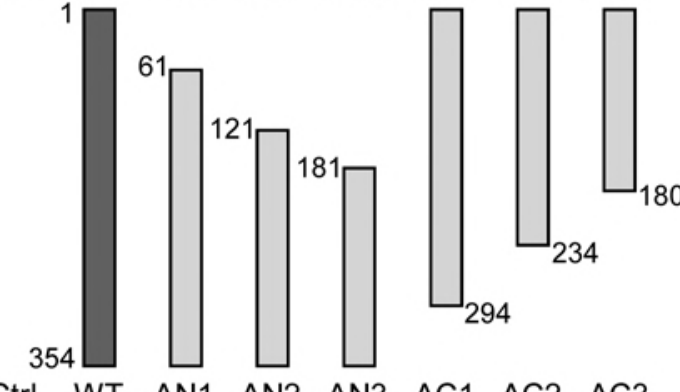

$\begin{array}{llllllll}\mathrm{Ctrl} & \mathrm{WT} & \Delta \mathrm{N} 1 & \Delta \mathrm{N} 2 & \Delta \mathrm{N} 3 & \Delta \mathrm{C} 1 & \Delta \mathrm{C} 2 & \Delta \mathrm{C} 3\end{array}$

Figure 3 
A

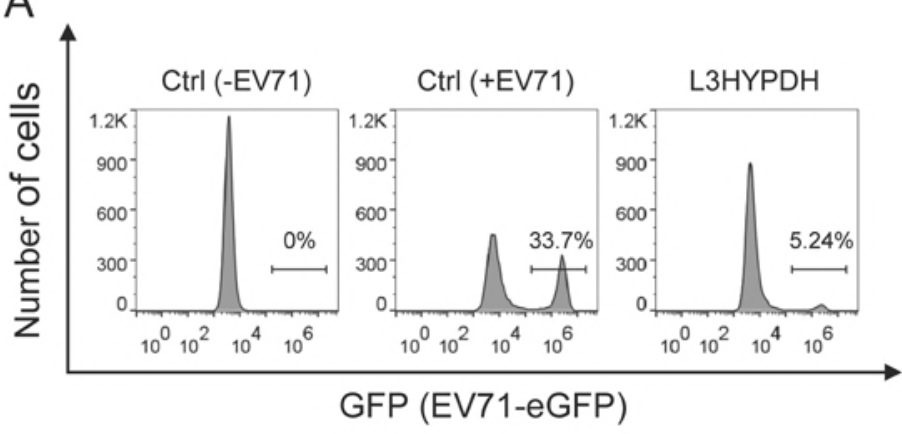

B



C

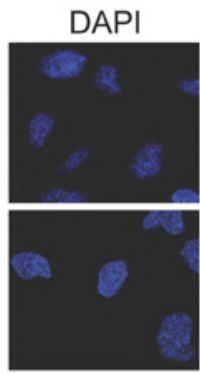

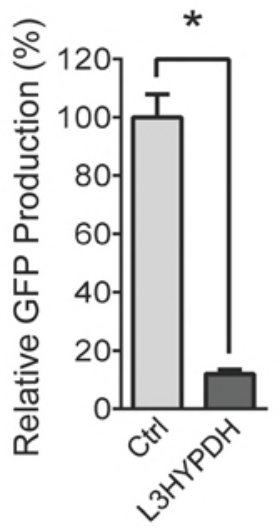

L3HYPDH

Merge

A

A

Ctrl



L3HYPDH

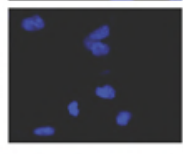

$\mathrm{B}$

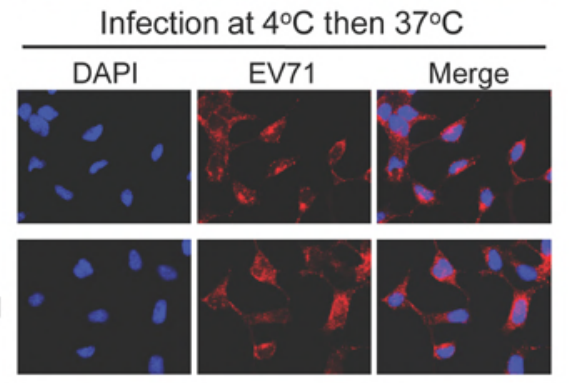

D

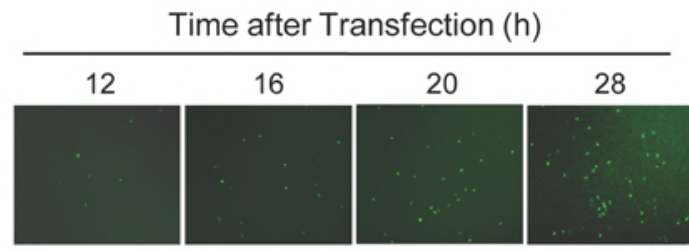

L3HYPDH
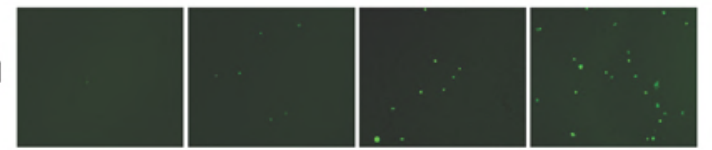

630
Infection at $4^{\circ} \mathrm{C}$

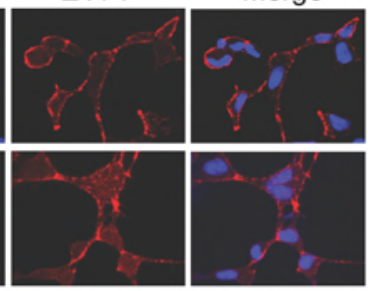

L3HYPDH

631

Figure 5

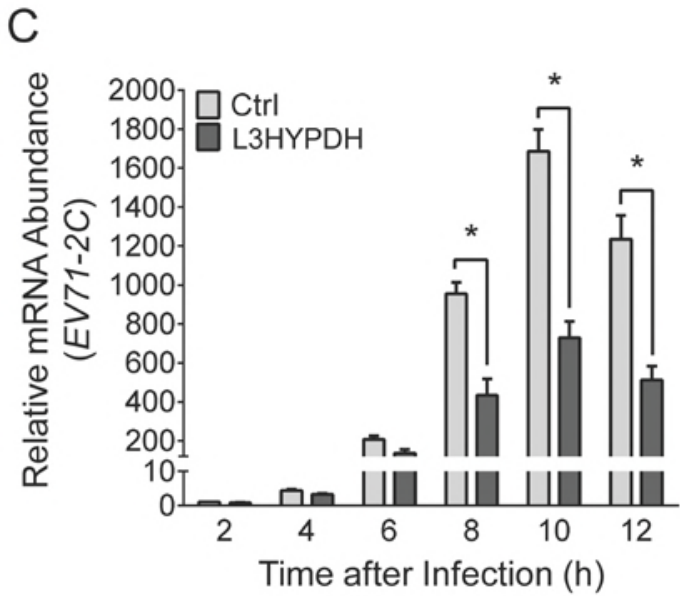

E



Time after Transfection (h) 
A
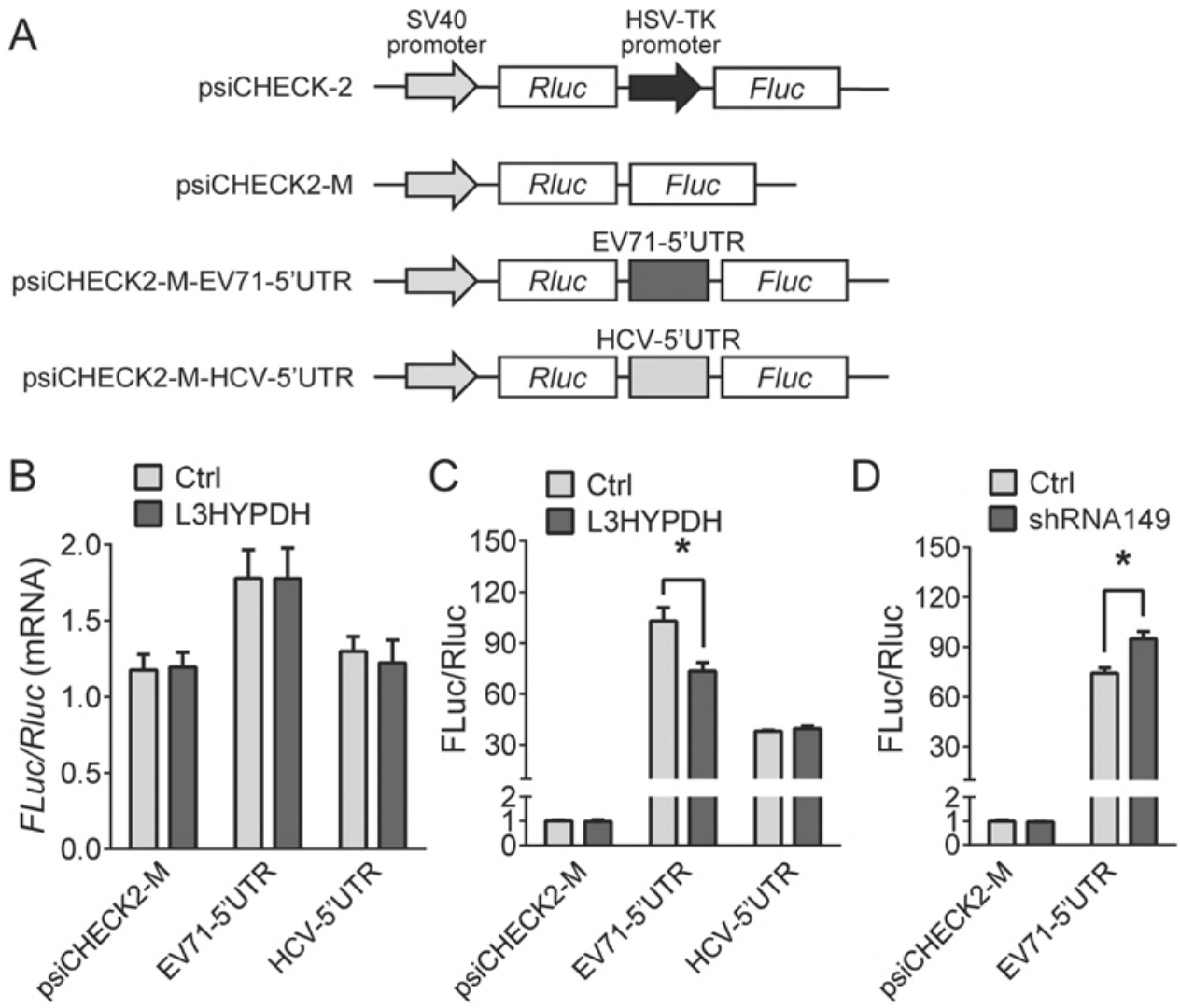

$E$

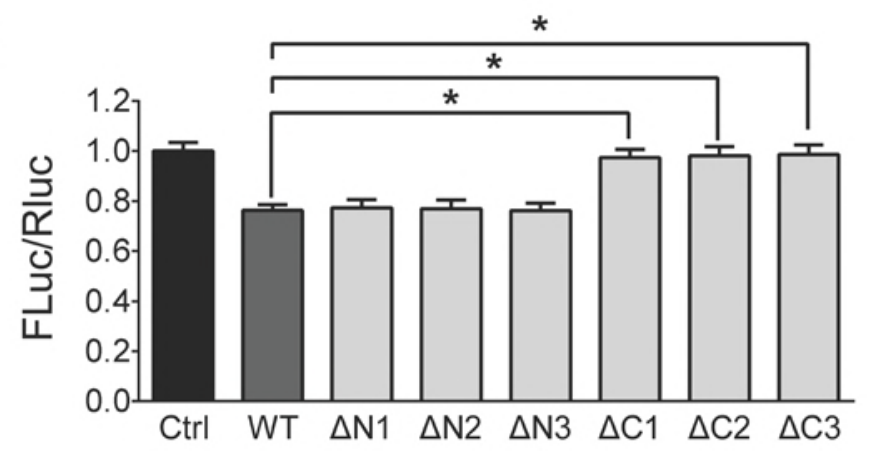

633

Figure 6 
Table S1 Primers used in this study

\begin{tabular}{|c|c|c|}
\hline Name & Sequence (5’-3’) & Target gene, usage \\
\hline L3HYPDH-F & CGGGATCCGCCACCATGGAGAGCGC ${ }^{\mathrm{a}}$ & \multirow{2}{*}{ LЗHYPDH } \\
\hline L3HYPDH-R & CACGCGGCCGCACTTGAGAAGAAATCCA & \\
\hline L3HYPDH-N1-F & CACGGTACCATGGTGCGGCGACGGCTCA & \multirow{2}{*}{ L3HYPDH $\Delta \mathrm{N} 1$} \\
\hline L3HYPDH-N-R & CACTCTAGACTGCACTTGAGAAGAAAT & \\
\hline L3HYPDH-N2-F & CACGGTACCATGGTGCCGGCGCCCCCTG & Pairing with L3HYPDH-N-R, L3HYPDHAN2 \\
\hline L3HYPDH-N3-F & CACGGTACCATGGGAAAGGTGATGGTGG & Pairing with L3HYPDH-N-R, L3HYPDH $\Delta$ N3 \\
\hline L3HYPDH-C-F & CACGGTACCATGGAGAGCGCGCTGG & \multirow{2}{*}{ L3HYPDH $\Delta \mathrm{C} 1$} \\
\hline L3HYPDH-C1-R & CACTCTAGACTCATCTGGTTCAGTTCC & \\
\hline L3HYPDH-C2-R & CACTCTAGACTTTCACTATCAGGATGA & Pairing with L3HYPDH-C-F, L3HYPDH $\Delta \mathrm{C} 2$ \\
\hline L3HYPDH-C3-R & CACTCTAGACTATGTCCAGGAACATCC & Pairing with L3HYPDH-C-F, L3HYPDH $\triangle \mathrm{C3}$ \\
\hline CHECK2-F & CACGCGGCCGCTCTAGGTTTAAA & \multirow{2}{*}{ Back-to-back primers, used for generating psiCHECK2-M } \\
\hline CHECK2-R & CACGTCGACATGGCCGATGCTAAGAACATTA & \\
\hline EV71-5’UTR-F & CACGCGGCCGCTTAAAACAGCCTGTGG & \multirow{2}{*}{ EV71-5’UTR } \\
\hline EV71-5’UTR-R & CACGTCGACGTTTAGCTGTGTTAAGG & \\
\hline HCV-5’UTR-F & CACGCGGCCGCGGCGACACTCCACCATAG & \multirow{2}{*}{ HCV-5’UTR } \\
\hline HCV-5’UTR-R & CACGTCGACGATGCACGGTCTACGA & \\
\hline QL3HYPDH-F & AGGAGTGACAGCC CGAATTG & \multirow{2}{*}{$L 3 H Y P D H$, used for qPCR } \\
\hline QL3HYPDH-R & CACATTTCGCTTCССТCACAG & \\
\hline QEV71-2C-F & TGTATGTCTCATTATCAGGGG & \multirow{2}{*}{ EV71 2C, used for qPCR } \\
\hline QEV71-2C-R & CCACCTGTTGCTTGTAACCGT & \\
\hline QRluc-F & ATAACTGGTCCGCAGTGGTG & \multirow{2}{*}{ Rluc, used for qPCR } \\
\hline QRluc-R & AGGCC GCGTTACCATGTAAA & \\
\hline
\end{tabular}




\begin{tabular}{|c|c|c|}
\hline QFluc-F & AGCACTTCTTCATCGTGGACCG & \multirow{2}{*}{ Fluc, used for qPCR } \\
\hline QFluc-R & GGCAGCTCGCCGGCATCGTCGT & \\
\hline H-QGAPDH-F & GAAGGTGAAGGTCGGAGT & \multirow[t]{2}{*}{ Human GAPDH, used for $\mathrm{qPCR}^{\mathrm{b}}$} \\
\hline H-QGAPDH-R & GAAGATGGTGATGGGATTTC & \\
\hline Vero-QGAPDH-R & GAAGATGGTGATGGGGCTTC & Pairing with H-QGAPDH-F, Monkey GAPDH, used for qPCR of the RNA from Vero cells \\
\hline
\end{tabular}

${ }^{\mathrm{a}}$ Restriction sites are underlined.

${ }^{\mathrm{b}}$ The primers against human GAPDH have been described elsewhere (Ng et al., 2002. Clinical Chemistry). All other primers used for the PCR assays are designed using the Primer-BLAST tool (https://www.ncbi.nlm.nih.gov/tools/primer-blast/) or acquired from Primer Bank (https://pga.mgh.harvard.edu/primerbank/). 


\section{Knockdown of L3HYPDH impairs anti-EV71 efficacy of IFN- $\alpha 2 b$}

IFN- $\alpha 2 b$ treatment combination with RNAi assay were performed to further determine if the endogenous L3HYPDH could suppress EV71 replication, 293A-SCARB2 cells were transfected with pSUPER-GFP-shRNA149, and the GFP-positive cells were sorted by FACS and divided into two parts for IFN- $\alpha 2 b$ treatment and mock-treatment, followed by EV71-MZ infection. RT-qPCR assay revealed that the IFN treatment caused a triple increase of the endogenous $L 3 H Y P D H$ mRNA level (Figure S1A) and about 40\% reduction in the EV71 RNA abundance in 293A-SCARB2 cells (Figure S1B), further validating the perspective that type I IFN is capable of inhibiting EV71 infection. When the expression of $L 3 H Y P D H$ was reduced by approximate $80 \%$ by RNAi, IFN- $\alpha 2 \mathrm{~b}$ treatment was less effective against EV71 replication and the viral RNA level increased from 0.6 to 0.78 (Figure S1A, S1B). Although the increase in viral yield was not significant, the results indicated that the $L 3 H Y P D H$ products play an irreplaceable role in the anti-EV71 action intrigued by IFN- $\alpha 2 \mathrm{~b}$, suggesting $L 3 H Y P D H$ as an important ISG possessing antiviral activity. 

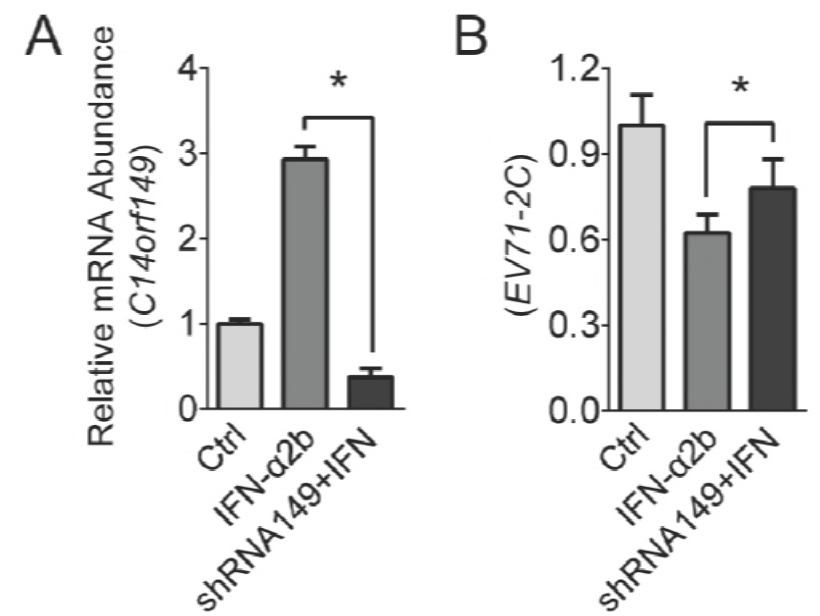

Fig S1. Effects of $L 3 H Y P D H$ knockdown on antiviral activity of IFN- $\alpha 2 b$ against

EV71. RT-qPCR analyses of the L3HYPDH (A) and EV71 2C (B) mRNA in 293A-SCARB2 upon depression of $L 3 H Y P D H$ expression by RNAi in the presence of IFN- $\alpha 2 b$. 293A-SCARB2 cells were seeded into a $10 \mathrm{~cm}$ dish and then transfected with $5 \mu \mathrm{g}$ of pSUPER-GFP-shRNA149 or pSUPER-GFP. After incubation for $24 \mathrm{~h}$, the GFP-positive cells were isolated using FACS and divided into two parts, one treated with $1000 \mathrm{IU} / \mathrm{ml}$ of IFN- $\alpha 2 \mathrm{~b}$, the other mock-treated with water as control, followed by EV71-MZ infection (MOI, 0.1). Eighteen hours post-infection, total RNAs were isolated and used for RT-qPCR measurement. The target mRNA level was normalized to that of GAPDH, and the relative value from the control cells was set as 1. The results are represented as mean $\pm \mathrm{SD}$ of three independent experiments. *, $P<0.05$. 